\title{
Sinusoidal Constriction and Vascular Hypertrophy in the Diabetes-Induced Rabbit Penis
}

Vivian Alves Pereira, Marcelo Abidu-Figueiredo, Marco Aurélio Pereira-Sampaio, Mauricio Alves Chagas, Waldemar Silva Costa, Francisco J. B. Sampaio

Urogenital Research Unit. State University of Rio de Janeiro (VAP, WSC, FJBS), Rio de Janeiro; Institute of Biology, Sector of Animal Biology, Rio de Janeiro Federal Rural University (MAF) Seropedica and Laboratory of Cellular and Extracellular Biomorphology, Department of Morphology, Federal Fluminense University (MAPS, MAC), Niterói, Rio de Janeiro, Brazil

\section{ABSTRACT}

Objective: To assess the morphological changes of penile vascular structures and the corpus cavernosum area in alloxan-induced diabetic rabbits.

Materials and Methods: Twenty male rabbits (2 months old) were divided into two groups with 10 rabbits each, the control group (CG) and the diabetic group (DG). The animals from DG received an intravenous injection of alloxan $(100 \mathrm{mg} / \mathrm{kg})$ to induce the diabetes. Ten weeks after the induction of diabetes, all animals were euthanized. Two fragments of the penile shaft were harvested and samples were processed and paraffin embedded. Sections $(5 \mu \mathrm{m})$ were cut and stained for histological and immunohistochemical markers.

Results: Nuclear protrusion toward the lumen, and cytoplasmic vacuolization were observed in the tunica intima of the dorsal artery of the penis in DG. The thicknesses of the tunica media increased significantly in DG ( $p=0.0350)$. It was also observed a significant increase in the area of the tunica media $(p=0.0179)$. There was no significant change in smooth muscle cell density in the tunica media of the dorsal artery of the penis $(p=0.0855)$. The collagen fiber pattern of the tunica adventitia of the dorsal artery of the penis was different between the control and diabetic groups. There was a significant decrease in the area occupied by the cavernous sinuses in DG ( $p=0.0013)$. Conclusion: Alloxan-induced diabetes mellitus in rabbits promotes important changes in penile vascular structures, thereby decreasing blood supply and affecting penile hemodynamics, leading to erectile dysfunction.

\section{ARTICLE INFO}

\section{Key words:}

Arteries; Diabetes Mellitus;

Penis; Rabbits

Int Braz J Urol. 2013; 39: 424-31

Submitted for publication:

July 07,2012

Accepted after revision:

December 14, 2012

\section{INTRODUCTION}

The penile erection results from blood pressure increases, relaxation of smooth muscle, and reduction of venous return (1). Smooth muscle cells, elastic system fibers, and collagen fibers are important penile structures involved in the erection process. These structures also give required rigidity to the penis when in a flaccid state $(2,3)$. Several studies have reported functional and morphological changes that take place during the erection process in different animal models $(4,5)$ and also in humans $(6,7)$.

Patients with diabetes mellitus (DM) have a high incidence of erectile dysfunction (ED). Several epidemiological studies have assessed the correlation between DM and ED (8). Evaluation of animal models demonstrated that neural (9) 
and vascular (10) changes seen with DM may be associated with ED. Vascular diseases such as microangiopathy, atherosclerosis, and hypertension are often observed in patients with DM. A relationship between these vascular conditions and the occurrence of morphological changes in the mesenteric artery $(11,12)$ and aorta in rats (13) has been demonstrated.

Although some morphological changes in penile elements have been described in rabbits with DM (3), there is no data regarding morphological changes in penile blood vessels associated with DM. The rabbit penis is, morphologically, classified as a vascular type and has a dorsal corpus cavernosum (CC) and a ventral corpus spongiosum (CS), which surrounds the penile urethra. Both CC and CS are covered by a dense connective layer, the tunica albuginea, which originates intra-cavernosum pillars or septa, mainly in the CC (14). There are pronounced resemblances between the rabbit and human penis in morphological, physiological, and neurological features $(5,15)$. Therefore, the rabbit penis is often used as an experimental model to assess ED $(5,14)$. The rabbit penis is a more suitable model than the rat penis, which is classified as a fibro-elastic type (4), with a penile bone inside and a penile protrusion during mating with little variation in its diameter (16).

The arterial vascularization of the penis in the New Zealand rabbit was shown to be supplied by the penile artery arising from the internal pudendal artery, and its branches, and the deep artery and dorsal artery of the penis (17). The dorsal artery of the penis (DAP) has a longitudinal path in the penis, with few variations, resulting in accurate transverse cuts by minimizing the bias in histomorphometric analysis. The DAP can be considered, from a histological perspective, as a representation of how DM and other disorders affect the penile arteries, as cited by Qiu et al. (18) and Kovanecz et al. (19). Additionally, the DAP is important for the homeostasis and function of penile tissues (19). Thus, the aim of this study was to assess the morphological changes in penile vascular structures and the area of the corpus cavernosum in diabetes-induced rabbits.

\section{MATERIALS AND METHODS}

Twenty New Zealand rabbits, 2 months old, weighing 1.5 to $2.0 \mathrm{Kg}$, were used in this study. The study was approved by the Ethics Committee in Animal Research at the State University of Rio de Janeiro (CEA 227/2008). All animals were housed in individual cages at room temperature. They were provided with a commercial rabbit feed (120 g/day) and water ad libitum. The animals were divided into two groups of 10 animals, the control group (CG) and the diabetic group (DG). All rabbits were anesthetized by using xylazine $(5 \mathrm{mg} / \mathrm{Kg}$ IM) and ketamine (20 mg/Kg IM). Animals from the diabetic group received an intravenous injection of alloxan monohydrate $(100 \mathrm{mg} / \mathrm{Kg})$ for the induction of diabetes (20), while the control group received an intravenous injection of the same volume of saline.

Blood samples were drawn from the marginal ear vein from all animals for glucose evaluation after a fasting period of 10 hours, and at 24,48 , and 72 hours post- diabetes induction and weekly thereafter until the end of the experiment. The measurement of serum glucose levels was made by using the One Touch Ultra Glucometer (Johnson \& Johnson Company, Rio de Janeiro, Brazil). Rabbits with serum glucose levels $126 \mathrm{mg} / \mathrm{dL}$ or above were considered diabetic. Ten weeks after the induction of diabetes, all animals were euthanized by an intravenous injection of high dose sodium thiopental.

\section{Histological procedures}

The penises were histomorphometrically assessed in both the control and diabetic groups. The area of the CC sinuses, thickness and area of the tunica media, and density of the smooth muscle cells of the DAP were measured and recorded. The tunica adventitia and the tunica intima of the DAP were qualitatively evaluated. Two fragments of the penile shaft were collected and fixed; one was placed in Bouin's fluid and another in 10\% buffered formalin ( $\mathrm{pH}$ 7.3). All samples were routinely processed and embedded in paraffin. Sections $(5 \mu \mathrm{m})$ were cut for use in immunohistochemistry and histological staining. 
Hematoxylin and eosin stain and Masson's trichrome stain were used for preliminary evaluation of the histological preparations. Picrosirius red stain, with a polarizing kit adapted to the microscope, was used to assess collagen birefringence in the DAP. The periodic acid-Schiff (PAS) technique was used to evaluate the endothelium (21).

\section{Immunohistochemistry}

The immunostaining was used to estimate the smooth cell density and area of the DAP and CC sinuses. The avidin-biotin-peroxidase method was used to identify smooth muscle cells. Briefly, the sections were dewaxed in xylene, hydrated in a decreasing series of ethanol into water, and washed in phosphate buffered saline (PBS) for 5 minutes. Sections were then treated at room temperature with a 3\% hydrogen peroxide solution in methanol to block endogenous peroxidase activity. Sections were then washed in PBS $(3 \times 5$ minutes) and incubated with $1 \%$ goat serum in a moist chamber for 30 minutes at $37^{\circ} \mathrm{C}$. Sections were then incubated with anti-alpha actin antibody (1:400, A-2547, Sigma-Aldrich Co, St Louis, MO, USA) in a moist chamber for 12 to 14 hours at $4^{\circ}$ C. Negative controls were incubated with PBS instead of the primary antibody. Samples of a well-known tissue, with the antigen, were used for the positive controls, as previously described (4). Finally, the sections were washed in PBS (3 $x 5$ minutes) and incubated with the biotinylated secondary antibody (Sigma-Aldrich Co, St Louis, MO, USA) at 1:100 in a moist chamber for $30 \mathrm{mi}-$ nutes at room temperature, washed and incubated with the $A B C$ complex (extravidin 1:100) for 30 minutes. The sections were then washed treated with a 393-diaminobenzidine tetrahydrochloride solution (Sigma-Aldrich Co, St Louis, MO, USA). The negative control was done by replacing the anti-smooth muscle a-actin antibody with PBS and no sign of staining was observed.

\section{Histomorphometry}

Selected images used for measurements and quantification were obtained using a light microscope (Olympus BX-41 coupled to a Sony CCD video camera), at magnifications of 4, 40, and 100 $\mathrm{x}$. Three sections from each animal were analyzed at different histological fields. Image $1.44 \mathrm{p}(\mathrm{Na}-$ tional Institute of Health, Bethesda, MD, USA) was used to quantify the area DAP wall. The cell counter.jar plug-in was used to count the smooth muscle cells of the tunica media of DAP and of the trabeculae of the CC.

The percentual of area occupied by cavernous sinuses was obtained by the difference between the CC area and the cavernous sinus area $\left(\mathrm{mm}^{2}\right)$. From these values we calculated the individual percentage of area occupied by the cavernous sinus, and then calculated the mean values for each group, in order to compare them.

\section{Statistics}

All results were expressed as mean \pm standard deviation (SD). Statistical analysis was performed using the Graphpad Instat software version 3.01 for Windows XP, (GraphPad Software Inc, San Diego, CA, USA). Student's t-test was used to test differences between the measurements from the control and diabetic groups, a value of $p$ $<0.05$ was considered statistically significant.

\section{RESULTS}

\section{Serum glucose}

After 72 hours from the intravenous injection of alloxan, serum glucose levels in rabbits reached $150 \mathrm{mg} / \mathrm{dL}$, and rabbits were then considered diabetic. In subsequent samples, the serum glucose levels increased in the diabetes-induced animals. In contrast, the mean serum glucose concentration in the control group remained at 79 $\mathrm{mg} / \mathrm{dL}$ throughout the experiment. At euthanasia, 10 weeks after the achievement of diabetes, the serum glucose levels of the diabetic group was approximately $350 \mathrm{mg} / \mathrm{dL}$. Therefore, rabbits in the diabetic group remained in a hyperglycemic state throughout the 10-week experimental period.

\section{Morphological changes in the DAP}

Nuclear protrusions toward the lumen and vacuolization of the cellular cytoplasm were observed in cells of the epithelium of the tunica intima in the DAP (Figures 1 and 2). The tunica media of the DAP was observed at high magnification to assess thickness and area (Figures 3 
Figure 1 - Tunica intima of the DAP of a control rabbit. Sections were stained with PAS stain, and images were taken at 1000x magnification. Scale bar: $10 \mu \mathrm{m}$.

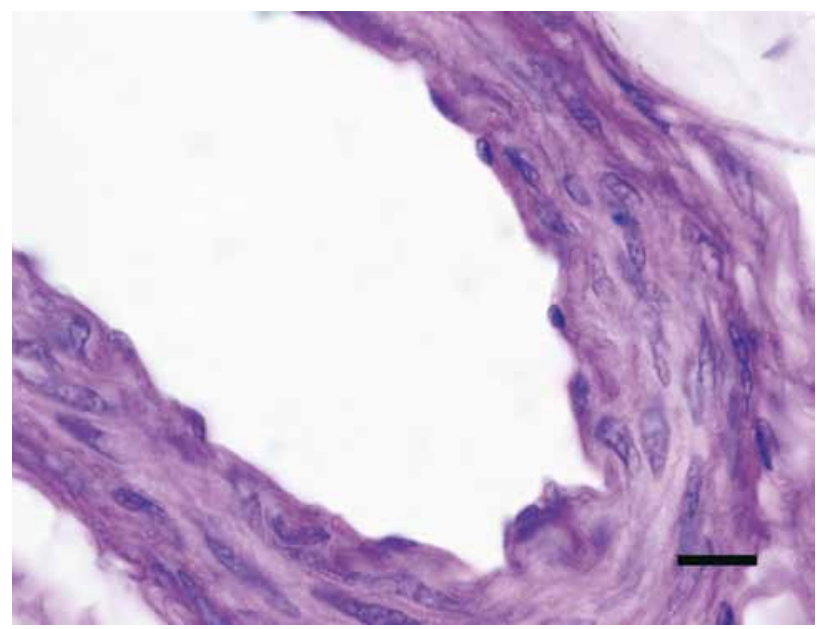

Figure 3 - Immunostaining of the smooth muscle in the tunica media of the DAP of rabbits from the control group. Sections were stained with the anti-alpha-actin antibody, and images were taken at $400 x$ magnification. Scale bar: $50 \mu \mathrm{m}$.

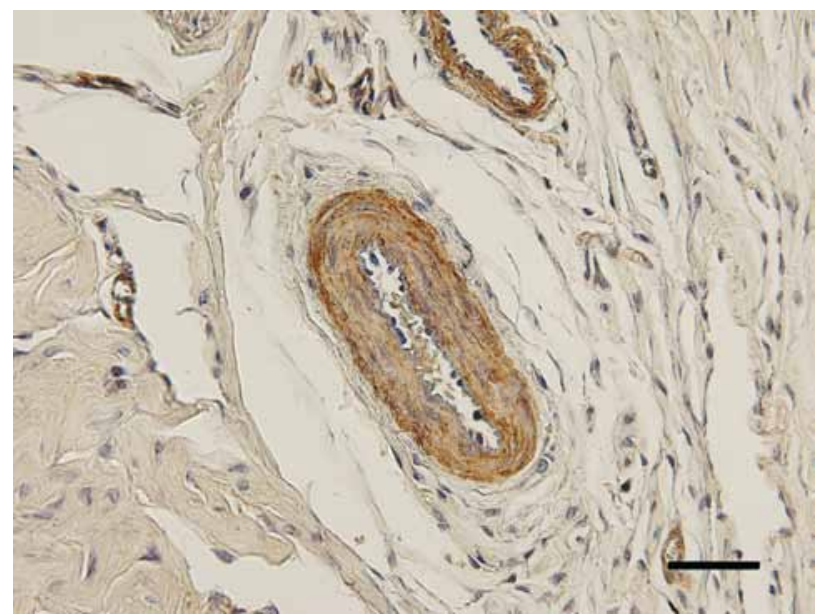

and 4). There were significant changes in the thickness and area of DPA wall (Table-1). The values of DPA wall thickness were $35.012 \pm 3.177 \mu \mathrm{m}$ in CG and $44.330 \pm 8.434 \mu \mathrm{m}$ in $\mathrm{DG}(\mathrm{P}=0.0350)$. The mean DPA wall area was $12070.675 \pm 2938.2 \mu \mathrm{m}^{2}$ and $18221.298 \pm 6861.9 \mu^{2}$ in control and diabetic groups, respectively $(\mathrm{P}=0.0179)$.

There was no significant change in smooth muscle cell density in the tunica media of the
Figure 2 - Tunica intima of the DAP of a diabetic rabbit, showing cytoplasmic vacuolization (arrows) and nuclear protrusions (arrow heads) in the endothelium. Sections were stained with PAS stain, and images were taken at 1000x magnification. Scale bar: $10 \mu \mathrm{m}$.

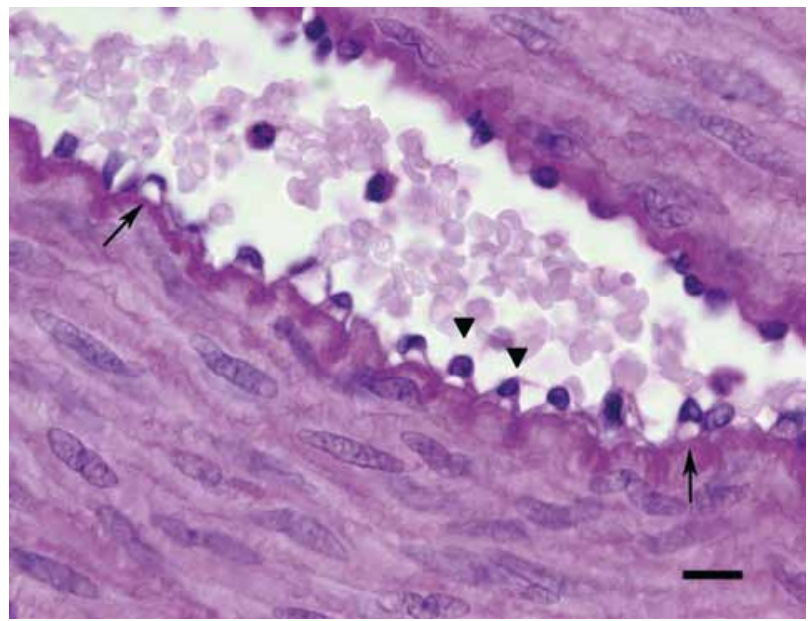

Figure 4 - Immunostaining of the smooth muscle in the tunica media of the DAP of rabbits from the diabetic group. Sections were stained with the anti-alpha-actin antibody, and images were taken at $400 x$ magnification. Scale bar: $50 \mu \mathrm{m}$.

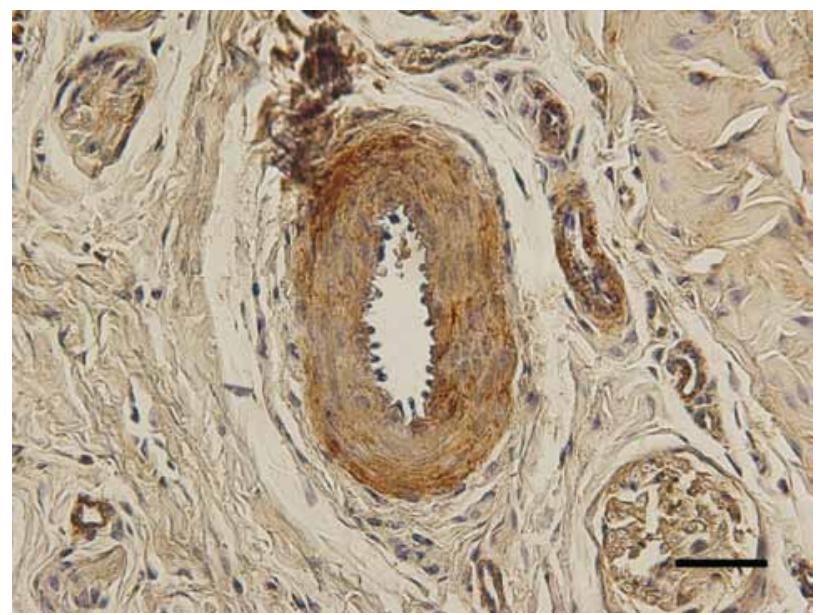

DAP between the groups. The ratio of nucleus per unit area was $0.007154 \pm 0.001954$ nuclei $/ \mu \mathrm{m}^{2}$ in CG and $0.004808 \pm 0.002069$ nuclei $/ \mu \mathrm{m}^{2}$ in the $\mathrm{DG}(\mathrm{P}=0.0855)$.

The collagen fibers of the tunica adventitia of the DAP were different between the control and diabetic groups. An orange birefringence and increased fiber thickness was observed in the control group. In the diabetic group, the birefrin- 
Table 1 - Morphological measurements of the DAP tunica media in control and diabetic rabbits. Data are presented as mean \pm SD.

\begin{tabular}{lccc}
\hline Parameter & Control group & Diabetic group & $P$ \\
\hline Thickness & $35.012 \pm 3.177 \mu \mathrm{m}$ & $44.330 \pm 8.434 \mu \mathrm{m}^{\star}$ & 0.0350 \\
Area & $12070.675 \pm 2938.2 \mu \mathrm{m}^{2}$ & $18221.298 \pm 6861.9 \mu \mathrm{m}^{2 *}$ & 0.0179 \\
Nuclear density & $0.007154 \pm 0.001954$ nuclei/ $/ \mathrm{m}^{2}$ & $0.004808 \pm 0.002069$ nuclei $/ \mu \mathrm{m}^{2}$ & 0.0855 \\
\hline
\end{tabular}

* Statistically significant $(p$ value $<0.05)$.

gence was greenish and the fibers were thinner (Figures 5 and 6).

\section{Morphometric analysis of CC sinuses}

There was a significant decrease in the mean area of the CC sinuses in the diabetic group compared to the control group (Figures 7 and 8). The values of the mean area in CG and DG were $60.76 \pm 7.883 \%$ and $37.93 \pm 9.986 \%$, respectively $(\mathrm{P}=0.0013)$.

However, there was no significant difference in the cellular density in the CC sinuses between the control and the diabetic groups.

\section{DISCUSSION}

The smooth muscle of both the CC and arterial wall of the DAP play an important role in penile erection (1). DM is associated with microand macrovascular diseases, which cause several morphological changes in the vascular wall (22). Wang et al. (23) demonstrated a high prevalence (> 75\%) of penile arterial insufficiency in diabetic men with erectile dysfunction, using duplex ultrasound after intracavernous injection of prostaglandin E1. Hyperglycemia in diabetic New Zealand rabbits has been shown to be associated with a reduction in the number of smooth muscle cells and increased cellular density when compared to normal rabbits, suggesting that hyperglycemia can lead to permanent changes in the dynamics of smooth muscle cell proliferation (24).

Diabetes can cause arterial wall thickening, as reported in the mesenteric artery, renal artery, and aorta $(11,12,25)$. However, this has not been previously described in penile arteries. The mechanism of arterial wall thickening associated
Figure 5 - Collagen arrangement in the tunica adventitia of the DAP of rabbits from the control group. Sections were stained with Picro Sirius red stain, and images were taken using polarized light at $400 x$ magnification. Scale bar: $50 \mu \mathrm{m}$.

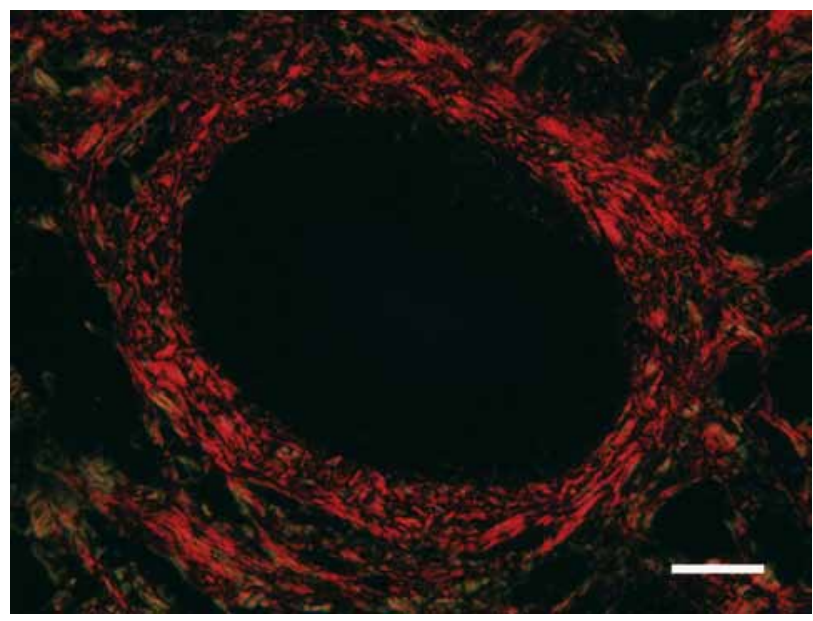

Figure 6 - Collagen arrangement in the tunica adventitia of the DAP of rabbits from the diabetic group. Sections were stained with Picro Sirius red stain, and images were taken using polarized light at $400 x$ magnification. Scale bar: $50 \mu \mathrm{m}$.

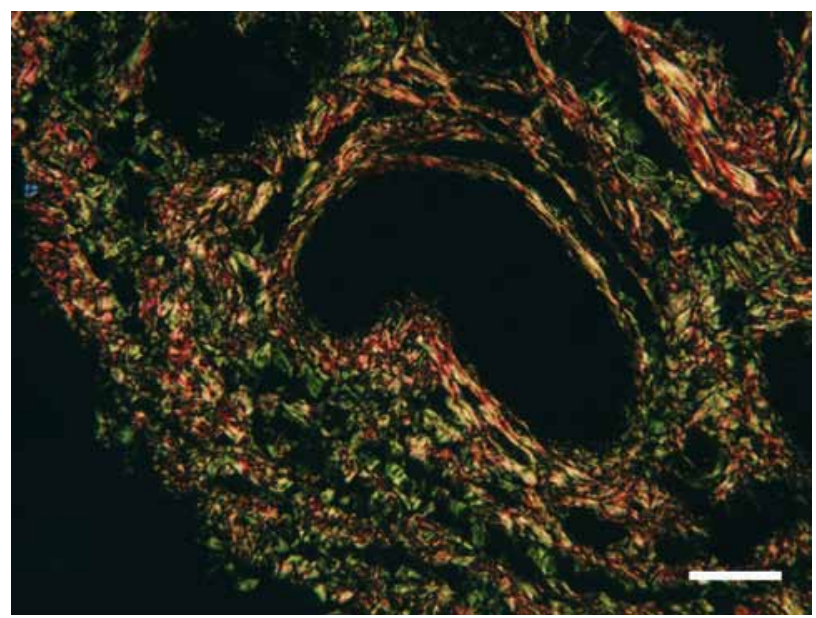


Figure 7 - Area of the CC sinuses of rabbits from the control group. Binarized image taken at 40x magnification. Scale bar: $200 \mu \mathrm{m}$.

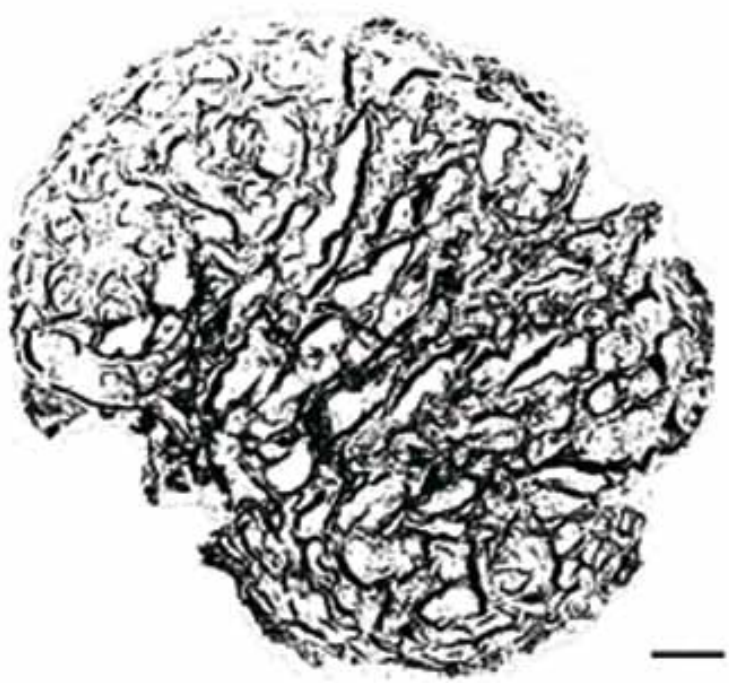

with DM is not clear, because smooth muscle cells of the tunica media may increase in size as a result of hyperplasia or hypertrophy. Hyperplasia of the neointima associated with DM has been shown to result from smooth muscle cell proliferation in the tunica media and subsequent migration to the tunica intima, leading to vascular stenosis (26). This migration process did not occur in the current study, and there was no disruption of the internal elastic layer. However, we demonstrated increased smooth muscle area and thickening of the DAP wall in diabetic rabbits, but with no change in the cellular density, suggesting hypertrophy of the smooth muscle cells in the tunica media. This finding agrees with Vranes et al. (11), who reported hypertrophy of the smooth muscle cells in the tunica media of thickened mesenteric arteries in diabetic rats. The relationship between DM and smooth muscle hypertrophy has also been demonstrated in the urinary bladder (27). Hypertrophic remodeling in the DAP tunica media can increase vascular resistance and impair the myogenic response, which is a key component of autoregulation of blood flow and stabilization of capillary pressure (22).

In this study, vascular remodeling associated with DM reached the tunica adventitia, chan-
Figure 8 - Area of the corpus cavernosum sinuses of rabbits from the diabetic group. Binarized image taken at 40x magnification. Scale bar: $200 \mu \mathrm{m}$.

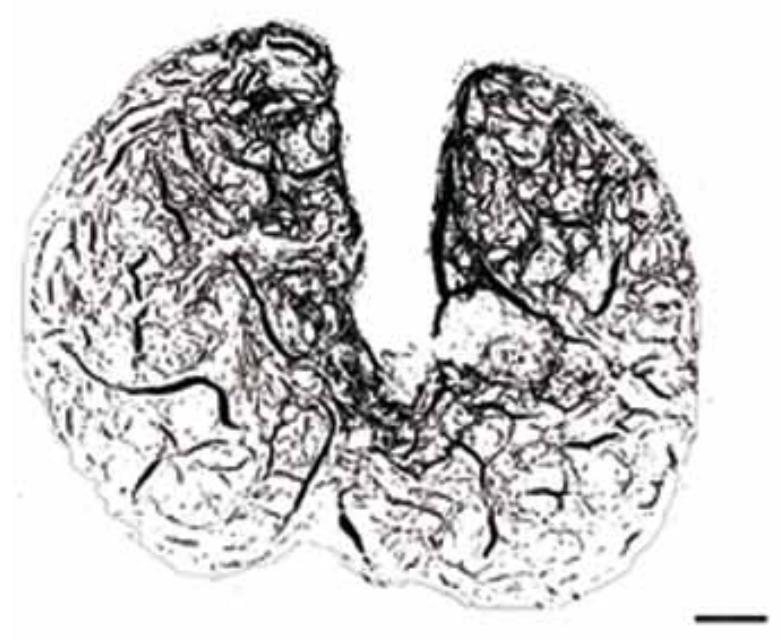

ging the pattern of collagen fibers and reducing their thickness from that seen in the control group (orange birefringence), to thinner fibers (greenish birefringence). Changes in the pattern of collagen fibers have been previously demonstrated in the mesenteric artery of streptozotocin-induced diabetic rats (11). Rearrangement of collagen fibers in the extracellular matrix, in both the wall of the DAP and periarterial region, suggests that it occurred simultaneously to vascular hypertrophy associated with DM. In this study, we also found nuclear protrusions toward the lumen and endothelial cytoplasmic vacuolization in the tunica intima. Hadcock et al. (25) have also reported cytoplasmic vacuolization in endothelial cells in the aorta of alloxan-induced diabetic rabbits. In cells from the umbilical cord, this vacuolization has been shown to be correlated with an increase in mitochondrial area (28). Mitochondrial and nuclear changes are indicators of the reversible injury mechanism, vacuolar degeneration (29). Chemically induced hyperglycemia may also cause oxidative stress leading to cellular vacuolization (25).

An increase in glucose promotes a small proliferative effect in the smooth muscle cells of coronary arteries. However, chronic hyperglycemia intensifies the response to growth factors 
such as platelet derived growth factor (PDGF) and transforming growth factor- $\beta 1$ (TGF- $\beta 1$ ) (30). The hypertrophy associated with DM in smooth muscle cells seen in the wall of the mesenteric artery could be associated with $\mathrm{Na} / \mathrm{H}$ equilibrium (31). The $\mathrm{Na} / \mathrm{H}$ channels of smooth muscle cells are sensitive to intracellular $\mathrm{pH}$ reductions that occur due to the ketoacidosis DM-related (29), which leads to higher activation of the $\mathrm{Na} / \mathrm{H}$ exchanger. $\mathrm{This} \mathrm{Na} / \mathrm{H}$ exchanger activation may be a response to higher amounts of glucose or to increased concentrations of the growth factors associated with DM, which can lead to hypertrophy (12).

The causes of vascular complications associated with DM are multifactorial, but glyco-oxidative stress has been shown to be a key factor among the several DM disorders (22). Advanced glycation endproducts (AGEs) play an important role in the decrease of vascular distensibility. The biochemical production of AGEs has been shown to be increased in DM, because of the chronic oxidative stress caused by hyperglycemia (32). In the current study, vascular impairment observed in the DAP associated with DM was not limited to the arterial wall. There was a decrease of $36 \%$ in the area of the CC sinuses, compared to control rabbits. This decrease in the sinus area can be explained by the increasing density of smooth muscle in the CC septa, thus enlarging the septal area (3).

In conclusion, experimentally induced DM by alloxan injection in rabbits resulting in 10 weeks of hyperglycemia causes important changes in the vascular structures of the penis, promoting alterations in the tunica intima, media, and adventitia, as well as significant decreases in the area of the CC sinuses. These changes decrease the blood supply and affect the hemodynamics of the penis, thus leading to ED.

\section{ABBREVIATIONS}

AGE: Advanced glycosilation end-product

CC: corpus cavernosum

CS: corpus spongiosum

DAP: dorsal artery of the penis

DM: diabetes mellitus

ED: erectile dysfunction

PBS: phosphate buffered saline
PDGF: platelet-derived growth factor

TGF- $\boldsymbol{\beta}$ : Transforming growth factor $\beta$

\section{CONFLICT OF INTEREST}

None declared.

\section{REFERENCES}

1. Lue TF, Tanagho EA: Physiology of erection and pharmacological management of impotence. J Urol. 1987; 137: 829-36.

2. Bastos AL, Silva EA, Silva Costa W, Sampaio FJ: The concentration of elastic fibres in the male urethra during human fetal development. BJU Int. 2004; 94: 620-3.

3. Abidu-Figueiredo M, Ribeiro IC, Chagas MA, Cardoso LE, Costa WS, Sampaio FJ: The penis in diabetes: structural analysis of connective tissue and smooth muscle alterations in a rabbit model. BJU Int. 2011; 108: 400-4.

4. Pinheiro AC, Costa WS, Cardoso LE, Sampaio FJ: Organization and relative content of smooth muscle cells, collagen and elastic fibers in the corpus cavernosum of rat penis. $J$ Urol. 2000; 164: 1802-6.

5. Bischoff E: Rabbits as models for impotence research. Int J Impot Res. 2001; 13: 146-8.

6. Papadoukakis S, Alamanis C, Mitropoulos D, Chountala A, Giannopoulos A: Morphologic findings and blood flow parameters of penile vasculature in patients with erectile dysfunction. World J Urol. 2004; 22: 285-8.

7. Costa WS, Carrerete FB, Horta WG, Sampaio FJ: Comparative analysis of the penis corpora cavernosa in controls and patients with erectile dysfunction. BJU Int. 2006; 97: 567-9.

8. Burke JP, Jacobson DJ, McGree ME, Nehra A, Roberts R0, Girman CJ, et al.: Diabetes and sexual dysfunction: results from the Olmsted County study of urinary symptoms and health status among men. J Urol. 2007; 177: 1438-42.

9. Zotova EG, Schaumburg HH, Raine CS, Cannella B, Tar M, Melman A, et al.: Effects of hyperglycemia on rat cavernous nerve axons: a functional and ultrastructural study. Exp Neurol. 2008; 213: 439-47.

10. Chitaley K: Type 1 and Type 2 diabetic-erectile dysfunction: same diagnosis (ICD-9), different disease? J Sex Med. 2009; 6(Suppl 3): 262-8.

11. Vranes D, Cooper ME, Dilley RJ: Cellular mechanisms of diabetic vascular hypertrophy. Microvasc Res. 1999; 57: 8-18.

12. Jandeleit-Dahm K, Hannan KM, Farrelly CA, Allen TJ, Rumble JR, Gilbert RE, et al.: Diabetes-induced vascular hypertrophy is accompanied by activation of $\mathrm{Na}(+)-\mathrm{H}(+)$ exchange and prevented by $\mathrm{Na}(+)-\mathrm{H}(+)$ exchange inhibition. Circ Res. 2000; 87: 1133-40. 
13. Peiró C, Angulo J, Rodríguez-Mañas L, Llergo JL, Vallejo $\mathrm{S}$, Cercas E, et al.: Vascular smooth muscle cell hypertrophy induced by glycosylated human oxyhaemoglobin. $\mathrm{Br} \mathrm{J}$ Pharmacol. 1998; 125: 637-44.

14. Maia RS, Babinski MA, Figueiredo MA, Chagas MA, Costa WS, Sampaio FJ: Concentration of elastic system fibers in the corpus cavernosum, corpus spongiosum, and tunica albuginea in the rabbit penis. Int $\mathrm{J}$ Impot Res. 2006; 18: 121-5.

15. Taub HC, Lerner SE, Melman A, Christ GJ: Relationship between contraction and relaxation in human and rabbit corpus cavernosum. Urology. 1993; 42: 698-704.

16. Beresford WA, Burkart S: The penile bone and anterior process of the rat in scanning electron microscopy. J Anat. 1977; 124: 589-97.

17. Ozgel 0 , Dursun N, Cengelci A, Ates S: Arterial supply of the penis in the New Zealand rabbit (Oryctolagus cuniculus L.). Anat Histol Embryol. 2003; 32: 6-8.

18. Qiu X, Fandel TM, Lin G, Huang YC, Dai YT, Lue TF, et al.: Cavernous smooth muscle hyperplasia in a rat model of hyperlipidaemia-associated erectile dysfunction. BJU Int. 2011; 108: 1866-72.

19. Kovanecz I, Nolazco G, Ferrini MG, Toblli JE, Heydarkhan $\mathrm{S}$, Vernet $\mathrm{D}$, et al.: Early onset of fibrosis within the arterial media in a rat model of type 2 diabetes mellitus with erectile dysfunction. BJU Int. 2009; 103: 1396-404.

20. Bozkurt NB, Pekiner C: Impairment of endothelium- and nerve-mediated relaxation responses in the cavernosal smooth muscle of experimentally diabetic rabbits: role of weight loss and duration of diabetes. Naunyn Schmiedebergs Arch Pharmacol. 2006; 373: 71-8.

21. Bancroft JD, Cook HC: Manual of Histological Techniques and Their Diagnostic Application. Edinburgh, Churchill Livingstone. 1994.

22. Rizzoni D, Rosei EA: Small artery remodeling in diabetes mellitus. Nutr Metab Cardiovasc Dis. 2009; 19: 587-92.
23. Wang CJ, Shen SY, Wu CC, Huang CH, Chiang CP: Penile blood flow study in diabetic impotence. Urol Int. 1993; 50: 209-12.

24. Alipui C, Ramos K, Tenner TE Jr: Alterations of rabbit aortic smooth muscle cell proliferation in diabetes mellitus. Cardiovasc Res. 1993; 27: 1229-32.

25. Hadcock S, Richardson M, Winocour PD, Hatton MW: Intimal alterations in rabbit aortas during the first 6 months of alloxan-induced diabetes. Arterioscler Thromb. 1991; 11: 517-29.

26. Varu VN, Ahanchi SS, Hogg ME, Bhikhapurwala HA, Chen A, Popowich DA, et al.: Insulin enhances the effect of nitric oxide at inhibiting neointimal hyperplasia in a rat model of type 1 diabetes. Am J Physiol Heart Circ Physiol. 2010; 299: H772-9.

27. Uvelius B: Detrusor smooth muscle in rats with alloxaninduced diabetes. J Urol. 1986; 136: 949-52.

28. Cester N, Rabini RA, Salvolini E, Staffolani R, Curatola A Pugnaloni $A$, et al.: Activation of endothelial cells during insulin-dependent diabetes mellitus: a biochemical and morphological study. Eur J Clin Invest. 1996; 26: 569-73.

29. Robbins SL, Cotran RS, Kumar V: Pathological basis of disease. Philadelphia, Saunders company. 2000; pp. 1-34.

30. Little PJ, Allen TJ, Hashimura K, Nigro J, Farrelly CA, Dilley RJ: High glucose potentiates mitogenic responses of cultured ovine coronary smooth muscle cells to platelet derived growth factor and transforming growth factor-beta1. Diabetes Res Clin Pract. 2003; 59: 93-101.

31. Dilley RJ, Farrelly CA, Allen TJ, Jandeleit-Dahm K, Cooper $\mathrm{ME}$, Morahan $\mathrm{G}$, et al.: Diabetes induces $\mathrm{Na} / \mathrm{H}$ exchange activity and hypertrophy of rat mesenteric but not basilar arteries. Diabetes Res Clin Pract. 2005; 70: 201-8.

32. Karasu C: Glycoxidative stress and cardiovascular complications in experimentally-induced diabetes: effects of antioxidant treatment. Open Cardiovasc Med J. 2010; 4: 240-56.

Correspondence address:

Prof. Waldemar Silva Costa

Av. 28 de Setembro, 87, fundos

Rio de Janeiro, RJ, 20562-030, Brazil

Tel.: + 5521 2868-8399

Fax: + 5521 2868-8399

E-mail: wscosta@gmail.com 